\title{
Stacked dielectric gratings for sub-wavelength surface field synthesis
}

\author{
C. J. Handmer, ${ }^{1,4}$ C. Martijn de Sterke, ${ }^{1,4}$ R. C. McPhedran, ${ }^{1,4, *}$ L. C. Botten, ${ }^{2,4}$ \\ M. J. Steel, ${ }^{3,4}$ and A. Rahmani ${ }^{2,4}$ \\ ${ }^{1}$ Institute for Photonics and Optical Science (IPOS), School of Physics, \\ University of Sydney, New South Wales 2006, Australia \\ ${ }^{2}$ Department of Mathematical Sciences, University of Technology, Sydney, New South Wales 2007, Australia \\ ${ }^{3} \mathrm{MQ}$ Photonics Research Centre and Department of Physics and Astronomy, \\ Macquarie University, Sydney, New South Wales 2109, Australia \\ ${ }^{4}$ Centre for Ultrahigh-Bandwidth Devices for Optical Systems (CUDOS), c/o School of Physics, University of Sydney, \\ NSW 2006, Australia \\ *Corresponding author: ross@physics.usyd.edu.au
}

Received June 23, 2010; revised September 5, 2010; accepted September 9, 2010;

posted September 24, 2010 (Doc. ID 130499); published November 11, 2010

\begin{abstract}
A method is developed to enhance the amplitudes of the non-propagating evanescent orders of resonant dielectric gratings. The origin of these resonances is analyzed in detail. The method relies on interactions between stacked gratings with different periods, and so a formalism is developed to model such stacks mathematically. In addition, a theoretical approach is developed to design gratings that enhance or blaze desired orders. These orders, controlled independently by incident fields from different angles, interfere and are optimized to produce steerable sub-Rayleigh field concentrations on a surface. These spots may function as a virtual scanning probe for non-invasive sub-Rayleigh microscopy. Optimization is conducted using a Monte Carlo Markov chain, and spots are generated which are both 1 order of magnitude narrower than the free space Rayleigh limit and robust to noise in the incident fields. (c) 2010 Optical Society of America
\end{abstract}

OCIS codes: $050.6624,050.1960$.

\section{INTRODUCTION}

The term "blazing" was introduced by Wood [1] to denote a technique for controlling the distribution of energy among propagating diffracted orders of a grating, made possible by the development of methods for accurately shaping the grating surface profile. After a century, it remains a standard technique. In general, the aim is to concentrate as much as possible the incident energy into one particular propagating order used for spectrometry or spectrography. Here we extend the usage of this term to encompass the intensity of evanescent (non-propagating) orders excited by diffraction gratings. Whereas Wood [1] and researchers were generally concerned with blazing propagating reflected orders, we will be interested in maximizing the intensities of a selected group of transmitted evanescent orders.

Conventional optical microscopy is fundamentally limited in resolution by the Rayleigh limit, which for radiation of wavelength $\lambda$ prevents information smaller than $\lambda / 2$ from propagating to the far field. Sub-wavelength field information is encoded in evanescent waves which, decaying rapidly away from the subject, cannot be imaged using a traditional microscope. The recovery of evanescent waves led to the popularization of exotic metamaterials [2] with the realization of their potential for microscopy beyond the Rayleigh limit. Such metamaterials utilize unusual permittivities and/or permeabilities to provide a focusing effect for both propagating and evanes- cent waves. While fabrication of a distortion-free optical superlens has proved extremely difficult, interest in imaging using evanescent waves has not died away. Yet if sub-wavelength information is to be recovered, some of it must be transformed into propagating waves and recovered in the far field. One proposed image forming system is the optical hyperlens [3]. Other approaches include stimulated emission depletion microscopy [4] and stochastic optical reconstruction microscopy [5], although these depend on the existence or introduction of fluorescing elements that allow the localization of sub-wavelength structures over a period of time.

A different approach to sub-wavelength microscopy uses regular variations in a substrate (a grating) to provide coupling between evanescent and propagating fields. The amplitude and phase in the far field are recorded, enabling computed tomography of the field at the surface [6-8]. Far-field optical diffraction tomography (FFODT) removes traditional constraints on resolution such as numerical aperture by utilizing strong coupling between evanescent and propagating waves. Strong coupling is vitally important, and demonstrations to date [9] have been limited by the absence of a theoretical apparatus for the enhancement of evanescent waves.

In a previous brief report [10] we introduced a general method for blazing evanescent orders. Using the example of lamellar gratings, we showed that the stacking of gratings with different periods allowed us to generate cus- 
tomizable sub-wavelength surface fields. In addition to providing the strong evanescent order coupling necessary for FFODT, we also presented a method by which narrow spots can be generated on the surface of the grating. These spots are up to a factor of 3 narrower than the Rayleigh limit for total internal reflection (TIR) $\lambda / 2 n_{H}$, where $n_{H}$ is the greatest refractive index present at the interface. By varying the incident field, the spots can be continuously scanned across the surface of the grating. Beyond simply providing strongly enhanced evanescent orders, our approach can thus generate a virtual scanning probe, eliminating mechanical interference with the subject, a considerable issue with other methods of subwavelength microscopy.

The aim of this paper is to explain first the analytical method used to model stacks of gratings and to explain how and why such structures can blaze evanescent orders and synthesize sub-wavelength surface fields. We present what we believe to be new results in support of the generality of the method, in addition to an expansion and explanation of the theoretical advances and optimization techniques employed. We consider only singly periodic gratings in this paper (for a recent paper discussing subwavelength spots on a doubly periodic grating, see Sentenac and Chaumet [11]). There should be advantages if doubly periodic gratings were incorporated in the systems studied, such as polarization insensitivity for normally incident light. However, the computational burden of an accurate calculation of the diffraction properties of stacks of doubly periodic dielectric gratings is considerably greater than that for singly periodic gratings, even when using sophisticated techniques such as the Fourier modal method [12]. This extra load would have precluded the extensive searches for optimal structures underlying the results presented here, particularly since each grating diffraction problem is characterized by six parameters rather than three (there being two periods and two depth parameters, plus two angles to characterize incidence rather than one). In a similar way, we choose to restrict our discussion to one polarization [that where the electric field of the incident wave is aligned along the grooves of the grating: transverse electric (TE) or $E_{z}$ polarization]. Our optimized designs are then restricted to TE polarization, with a separate optimization being necessary for the transverse magnetic (TM) case. However, we stress that our design procedure is based on kinematic and Fourier arguments, which are general and apply equally well to singly and doubly periodic gratings, irrespective of the choice between efficiency-extremal directions of the polarization of light [13].

We begin with an outline of the modal method for lamellar gratings in Subsection 2.A, and then discuss general properties of single resonant dielectric gratings in Subsection 2.B, explaining how Fabry-Perot resonance of higher order grating modes leads to blazed evanescent orders. In Subsection 2.C we explain and justify the generalization of the modal method to gratings with different periods, while in Subsection 2.D we explain the rationale of stacking gratings with a particular period ratio and develop a Fourier model as a heuristic shortcut to first order optimization of the transmitted spectrum. In Section 3 we explain fully the optimization of a multi-dimensional
( 100) parameter space using a Monte Carlo Markov chain (MCMC), demonstrating and discussing results that go beyond our previous report [10] in Section 4. Following these new results we present a possible configuration for a system that can perform microscopy directly using a scanned sub-wavelength synthesized spot.

\section{THEORETICAL DEVELOPMENTS}

\section{A. Modal Method Summary and Description}

In this subsection, we derive rigorous techniques for modeling the propagating of fields through two-dimensional (2D) dielectric gratings. This will serve as the basis for analyzing the excitation of evanescent orders and the advantages obtained by combining multiple gratings. We begin with the diffraction grating equation

$$
k_{x p}=k_{0} \sin \theta_{0}+2 \pi p / d, \quad k_{y p}=\left(k_{0}^{2}-k_{x p}^{2}\right)^{1 / 2},
$$

where $k_{0}=2 \pi / \lambda, d$ is the period, and $k_{x p}$ and $k_{y p}$ are the $x$ and $y$ components of the wavevector $\mathbf{k}_{p}$ of the order $p$ plane wave with spatial phase variation $\exp \left(i k_{x} x+i k_{y} y\right)$. This equation is a generalization of Fraunhofer's grating equation $\sin \theta_{p}=\sin \theta_{0}+p \lambda / d$ and provides the foundation for a unified description of propagating and evanescent grating orders. When $|p|$ is large, $k_{x p}^{2}>k_{0}^{2}$, which causes $k_{y p}$ to be imaginary. The sign of the square root is chosen to prevent fields diverging away from the surface. Instead, the fields are evanescent, as they decay exponentially in the $y$ direction away from the surface of the grating as seen in Fig. 1. For $|p|$ sufficiently large, Eq. (1) takes the form $k_{y p} \sim i\left|k_{x p}\right| \approx 2 i \pi|p| / d$, independent of wavelength. This suggests the possibility of sub-Rayleigh resolution if we can excite these large $|p|$ orders. As $k_{y p}$ $\sim i\left|k_{x p}\right|$, evanescent waves have similar length scales in both the $x$ and $y$ directions, leading to an essentially semicircular profile. Unlike propagating waves, evanescent waves are discrete and localized.

As evanescent waves do not carry energy away from the surface of the grating, their amplitudes are not limited by conservation of energy and can potentially achieve amplitudes many times greater than ambient propagating fields. However, the kinematic diffraction grating equation does not predict the amplitudes of grating orders generated in transmission and reflection. For this a more involved calculation is required. We use the modal method for one-dimensional lamellar dielectric gratings [14], as it is a rigorous semi-analytical approach allowing a degree of physical insight as well as computational efficiency. For convenience, we examine only the TE/ $E_{z}$ polarization; however, generalizations for nearly every case

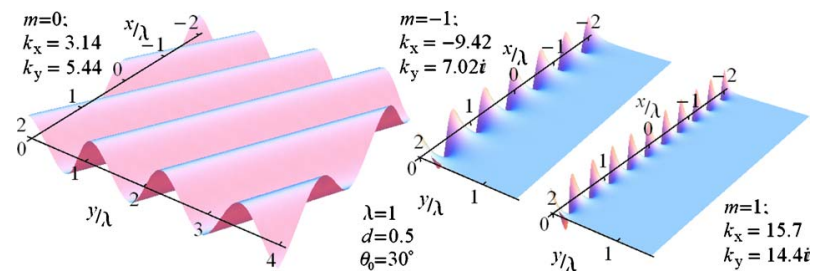

Fig. 1. (Color online) Central three plane-wave orders generated by a grating demonstrating propagation and evanescence. The grating occupies the region $y<0$ and has period $d=\lambda / 2$. Wavelength $\lambda=1$, angle of incidence $\theta_{0}=30^{\circ}$. 
of physical interest, including higher dimensionalities, both polarizations, composite structures, metallic inclusions, substrates, and off-axis incidence exist [15-18].

A lamellar grating is a $2 \mathrm{D}$ structure composed of alternating rectangles of different media (Fig. 2). In our work, the structure is typically made of $\mathrm{Si}\left(n_{\mathrm{Si}}=3.41\right)$ and $\mathrm{SiO}_{2}$ $\left(n_{\mathrm{SiO}_{2}}=1.46\right)$ and is a few microns thick. The modal method, which is similar to the rectangular potential Kronig-Penney model used in solid-state physics [19], is based around the calculation of Fresnel scattering matrices for interfaces between free space plane-wave orders and confined grating modes.

A full derivation of the modal method is given in several papers [14-16], so here we give a summary and quote relevant results as necessary. The method can be broken into several stages. First, the electric field in each region is decomposed into complete basis sets of either plane waves or grating modes, as explained in Appendix A. Second, the expansion of the field bases in each region is matched at the interface, and the change of basis matrix $\mathbf{J}$ is derived. Now that the fields at the free space-grating interface can be freely expressed and related in either basis, the mode-order Fresnel scattering matrices are derived, as shown in Appendix B. Appendix C describes how Fresnel scattering matrices for each interface are combined to create a scattering matrix representing the entire lamellar grating, which functions as a Fabry-Perot interferometer. The stacking of multiple gratings is a generalization of this procedure and is fully explained in Subsection 2.C.

In summary, the field in the grating is expressed as a sum of confined modes each of which is characterized by the horizontal propagation parameters $\gamma_{1}$ and $\gamma_{2}$ (defined in Appendix A) for each region of the grating (unit cell shown in Fig. 2) and a vertical propagation parameter $\beta$, indexed by $n$. Six of these modes are illustrated in Fig. 3 for one particular two-component grating. Likewise, the field in vacuum is composed from a discrete set of planewave orders characterized by the spatial frequency components $\alpha_{p}=k_{x p}$ and $\chi_{p}=k_{y p}$. Each field decomposition is a complete orthonormal set, so there exists a mode-order (or order-mode) change of basis matrix $\mathbf{J}$ with which complex amplitudes at the grating-free space interface can be reexpressed in terms of either basis. $\mathbf{J}$ has dimensions of $N_{p} \times N_{n}$, where $N_{p}$ and $N_{n}$ represent truncation limits for

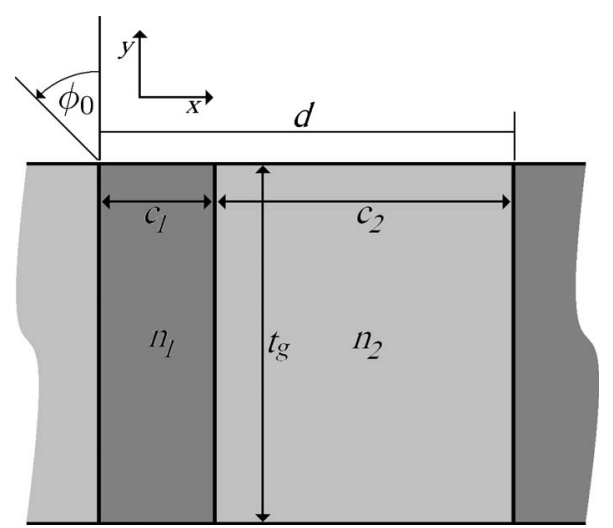

Fig. 2. Diagram of a unit cell of a lamellar grating, showing geometrical factors and regions of different refractive indices. orders and modes, respectively. As such it needs not be square, but for the minimization of truncation error it is usually kept at least close to square. For convenience we introduce spatial scale factors $\boldsymbol{\beta}=\operatorname{diag}\left\{\beta_{n}\right\}$ (for modes) and $\chi=\operatorname{diag}\left\{\chi_{p}\right\}$ (for orders) which combine with $\mathbf{J}$ to form

$$
\begin{aligned}
& \mathbf{A}=\boldsymbol{\chi}^{1 / 2} \mathbf{J} \boldsymbol{\beta}^{-1 / 2}, \\
& \mathbf{B}=\boldsymbol{\beta}^{-1 / 2} \mathbf{J}^{\dagger} \boldsymbol{\chi}^{1 / 2},
\end{aligned}
$$

from which the complex Fresnel scattering matrices for reflection or transmission at either interface are

$$
\begin{gathered}
\mathbf{R}_{12}=(\mathbf{A B}+\mathbf{I})^{-1}(\mathbf{A B}-\mathbf{I}), \\
\mathbf{R}_{21}=(\mathbf{B A}+\mathbf{I})^{-1}(\mathbf{I}-\mathbf{B A}), \\
\mathbf{T}_{12}=2 \mathbf{B}(\mathbf{A B}+\mathbf{I})^{-1}, \\
\mathbf{T}_{21}=2 \mathbf{A}(\mathbf{B A}+\mathbf{I})^{-1} .
\end{gathered}
$$

Incorporating a complex diagonal matrix $\mathbf{P}=\exp \left(i \boldsymbol{\beta} t_{g}\right)$ to account for vertical separation $t_{g}$ of two grating/free space interfaces to construct a grating, the overall reflection and transmission scattering matrices are

$$
\begin{gathered}
\mathbf{R}=\mathbf{R}_{12}+\mathbf{T}_{21} \mathbf{P} \mathbf{R}_{21} \mathbf{P}\left(\mathbf{I}-\mathbf{R}_{21} \mathbf{P} \mathbf{R}_{21} \mathbf{P}\right)^{-1} \mathbf{T}_{12}, \\
\mathbf{T}=\mathbf{T}_{21} \mathbf{P}\left(\mathbf{I}-\mathbf{R}_{21} \mathbf{P R} \mathbf{R}_{21} \mathbf{P}\right)^{-1} \mathbf{T}_{12} .
\end{gathered}
$$

\section{B. Phenomenology of Evanescent Order Blazing with a Single Grating}

The (I- $\left.\mathbf{R}_{21} \mathbf{P} \mathbf{R}_{21} \mathbf{P}\right)$ matrix is responsible for resonant behavior, as satisfying the Fabry-Perot condition for any propagating mode causes this term to become close to singular. Physically, Fabry-Perot resonances and corresponding conditions exist for every vertically propagating mode in the structure. Some of these modes couple very poorly to propagating orders and thus have very high finesse. When excited by incoming plane waves on resonance, they induce large enhancements of the transmitted and reflected evanescent orders. This is well illustrated by combining variation in both grating thickness and incident angle as seen in Fig. 4 (for the same grating parameters as in Fig. 3), which is a density plot of the amplitudes of the transmitted propagating [(a) $m=0$ ] and near evanescent [(b) $m=1$ ] orders. Figure 4(a) shows that the overall trend of a uniform dielectric slab toward greater reflection at glancing incidence is interrupted by several series of anomalies in which the reflectance rapidly oscillates between 0 and 1 before returning to its background trend. For gratings with narrow ridges these anomalies are naturally interpreted as grating-assisted coupling to slab waveguide modes. In the corresponding figure [Fig. 4(b)] for the evanescent order $m=1$, enhancements coincide with the anomalies seen in the propagating amplitude, demonstrating the evanescent order blazing that we are seeking. As exciting evanescent orders is a key to generating sub-wavelength field structures, a complete understanding of these resonances is central to our approach.

These grating anomalies are illustrated in Figs. 5 and 6 , which show the amplitude of the central-most grating 

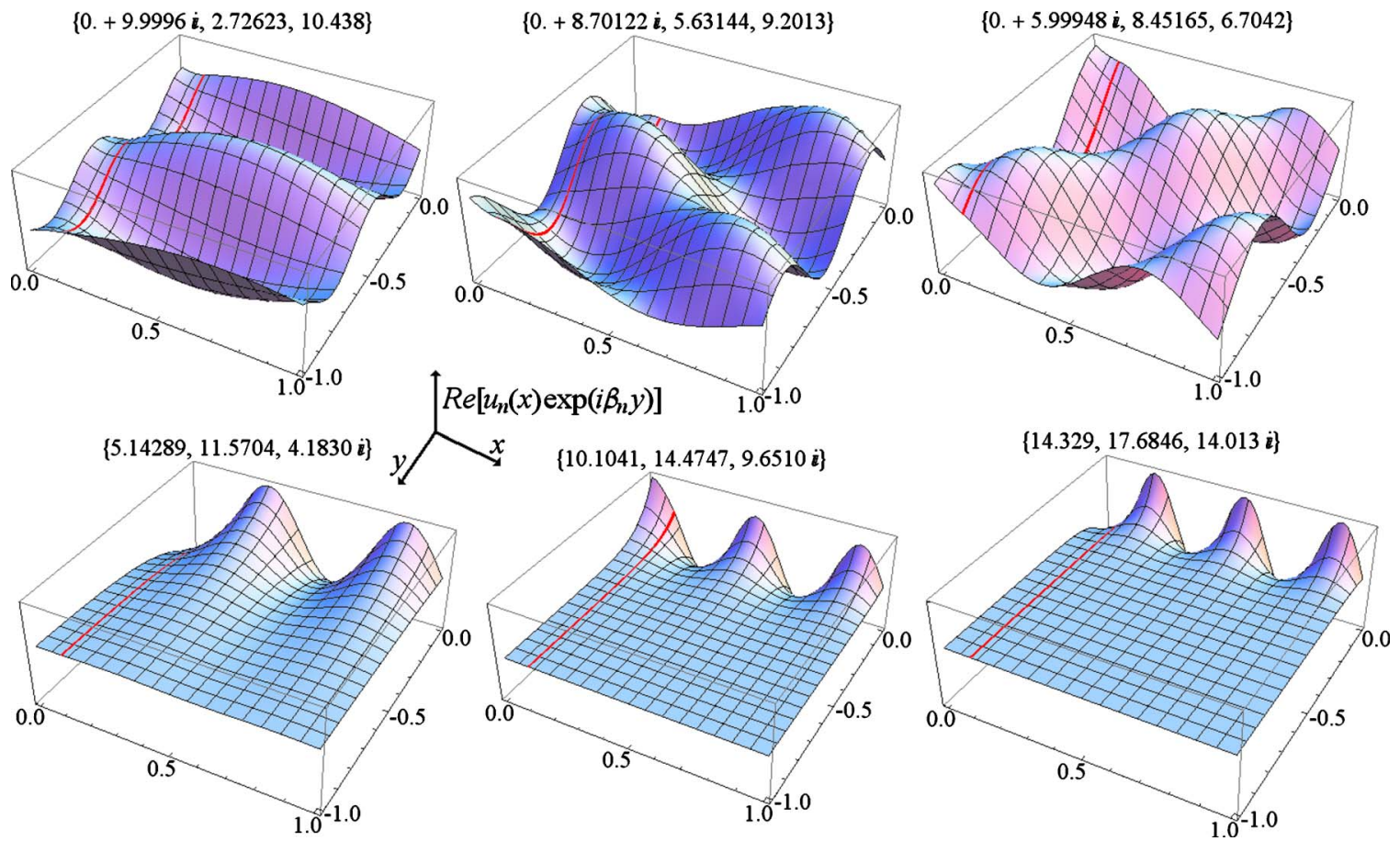

Fig. 3. (Color online) Plot of $\mathcal{R}\left[u_{n}(x) \exp \left(i \beta_{n} y\right)\right]$ for $n=\{1,2, \ldots, 6\}$ or the fields of the first six grating modes across period. As before the grating occupies $y<0$, has a period $d=1$, and a duty cycle $c_{1} / d=0.1$. The thicker solid line delineates the boundary between $n=1.0$ and $n=3.61$ in this particular example. Numbers above each graph give $\left\{\gamma_{1}, \gamma_{2}, \beta\right\}$ for each mode, emphasizing the propagating nature of the upper three modes and the evanescent nature of the lower three modes.

orders as a function of the angle of incidence. These figures clearly demonstrate distinct resonances and corresponding evanescent field blazing as the incident angle is varied. Additionally, the structure of the anomalies identify them as Fano resonances; a unique fingerprint for the higher order Fabry-Perot resonances occurring in the structure. Fano resonances are a generalization of Lorentzian resonances wherein a quickly varying resonance is coupled to a non-zero and slowly varying background. In this case, the presence of multiple vertically propagating modes in the grating provides the multiple channels necessary to produce Fano resonances. The fundamental grating mode couples strongly to propagating plane-wave orders and forms the slowly varying background, while higher order grating modes that couple to evanescent orders form the narrow high $Q$ resonances [20].

Given that only the incident angle can be varied on a fabricated structure, our analysis uses a fixed grating
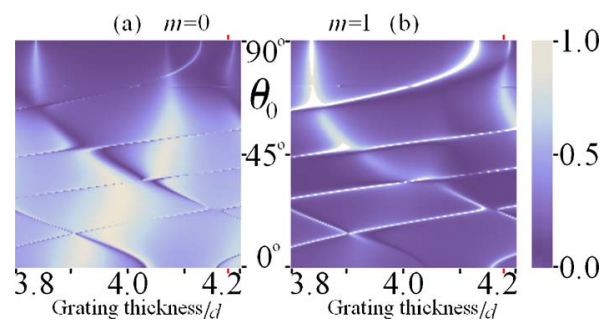

Fig. 4. (Color online) Density plot of the amplitude of the transmitted propagating $(m=0)$ and first evanescent $(m=1)$ orders as a function of incident angle and primary grating thickness (measured here in multiples of the grating period $d$ ). Chosen optimal thickness is $t_{g}=4.177 d=3.08 \mu \mathrm{m}$. The other grating parameters are the same as in Fig. 3. thickness. With a unit amplitude field (of constant phase) in a set of 200 calculated incident angles $\left[1^{\circ}, 89^{\circ}\right]$, data for a quasi-continuous spectrum of order amplitudes may be calculated for both reflection and transmission. The transmission spectrum of a single resonant grating is shown in Fig. 6. Provided the structure has left-right symmetry, the spectrum can be mirrored to $\left[-89^{\circ},-1^{\circ}\right]$ for no additional effort. This figure captures a lot more information than Fig. 5. For instance, the solid line in Fig. 5 refers to the part of Fig. 6 in transmission between zero and $k_{0}$. Strong evanescent field enhancement or blazing is present in the ranges $k_{x} \in\left[-n_{H} k_{0},-k_{0}\right]$ and $k_{x}$

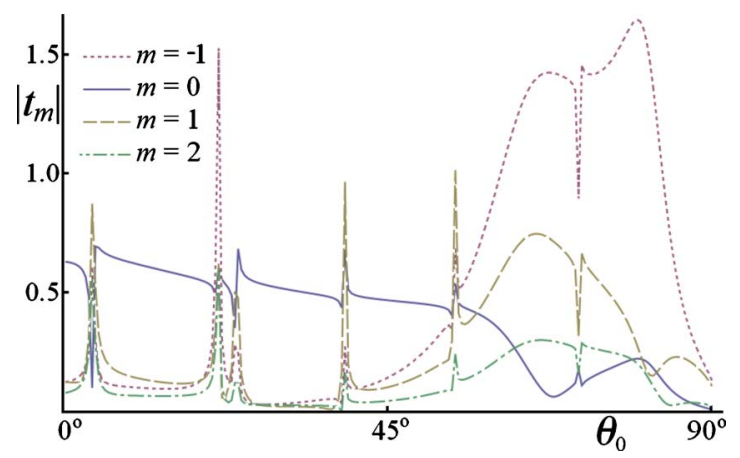

Fig. 5. (Color online) Line plot showing amplitude in transmission of central orders for the stacked grating of fixed thickness shown in Fig. 7 as a function of incident angle $\theta_{0}$. The solid line represents the propagating transmitted amplitude $(m=0)$, which trends downward through grazing incidence, interrupted by grating anomalies in the form of Fano resonances, signaling the enhancement of corresponding evanescent orders $(m=-1,1,2)$. The grating has period $d=0.738 \mu \mathrm{m}$, thickness $t_{g}=3.08 \mu \mathrm{m}$, and duty cycle of $10 \%$ for the low index component. 




Fig. 6. (Color online) Absolute value of the incident (inset) and transmitted spectrum from the $\mathrm{Si} / \mathrm{SiO}_{2}$ grating shown at right. The grating has period $d=0.738 \mu \mathrm{m}$, thickness $t_{g}=3.03 \mu \mathrm{m}$, and duty cycle of $10 \%$.

$\in\left[k_{0}, n_{H} k_{0}\right]$, which manifests as a region of sharp peaks on either side of the central propagating channel.

As can be seen in Figs. 5 and 6, a typical single grating produces many grating resonances at different incident angles, which are responsible for exciting evanescent orders with $k_{x}$ values in the ranges $\left[-n_{H} k_{0},-k_{0}\right]$ and $\left[k_{0}, n_{H} k_{0}\right]$, increasing the potential resolving power by a factor of $n_{H}$. In the following, we use this to improve the synthesis of narrow field spots. For our calculations of synthesized surface fields, incident fields are configured to excite every resonance found in spectra of the type shown in Fig. 5. Peaks are identified and the grating thickness is chosen automatically using a simple peakfinding search algorithm over the full range of $\left(-90^{\circ}, 90^{\circ}\right)$. This typically translates to between 30 and 60 incident angles (and thus up to 120 scalar variables in amplitude and phase) to have complete control over the blazed fields. Note that the search algorithm includes peaks in higher $|m|$ orders not shown in Fig. 5, which sometimes do not coincide perfectly with the more powerful resonances. Such resonances are typically very narrow, and may not be completely resolved in Fig. 6 or Fig. 5. Nonetheless their existence, as grating anomalies, is a well known phenomenon [21,22], visible via a Fano resonance signature present in the propagating fields. This signature (and the corresponding resonance) is strongest when the grating exhibits left-right symmetry and generates only one propagating order [23]. While this result can be derived from boundary conditions and energy conservation, a more intuitive reason is that multiple propagating orders undermine the independence of blazed evanescent orders by decreasing the range of incident angles that correspond independently to a particular set of unique orthogonal orders. That is, for some range of angles, a given set of evanescent orders couples to two (or more) propagating orders, undermining efficiency. Vice versa, two independent incident fields from either angle will excite the same set of evanescent orders, which is a waste of the limited $\left(-90^{\circ}, 90^{\circ}\right)$ range of possible incident angles. For this reason gratings used in this paper have a period that is slightly less than half the wavelength of the incident light and so have only a single propagating order for each incident angle.

C. Modal Method Generalization for Stacked Gratings A single resonant grating made of $\mathrm{SiO}_{2}$ and silicon can blaze evanescent orders, manifesting as sharp peaks in
Fig. 6. In the case of lamellar gratings, blazed evanescent orders are driven by propagating grating modes and are cut off when $\left|k_{x}\right|>n_{H} k_{0}$ as shown in Fig. 6. This cutoff corresponds to the immersion Rayleigh limit, which is the limit of resolution obtainable through TIR. In TIR, field variation due to a propagating wave in a dielectric is also present in the adjacent area of free space due to field continuity across the boundary. In free space, however, field variations that correspond to a wave propagating in a dielectric are too rapid to propagate, and the transverse component of the spatial frequency becomes imaginary. In this way, TIR gives rise to evanescent fields (as seen in frustrated TIR) but also imposes limits on the resolution obtainable with a single grating, as evanescent waves with $\left|k_{x}\right|>n_{H} k_{0}$ can only be generated very weakly by propagating waves in the dielectric. Despite this, resonant gratings are better than just TIR in at least one respect, as blazing greatly increases near evanescent order amplitudes.

What is needed to extend evanescent order blazing beyond near evanescent orders is a grating design that can take the energy present in blazed near evanescent orders (orders for which $\left|k_{x}\right|<n_{H} k_{0}$ ) and scatter it to more confined (spatially) far evanescent orders. Gratings with sufficiently small periods have only a single (bound) propagating grating mode. As they lack higher order propagating modes, they necessarily lack the high $Q$ grating resonances which blaze evanescent orders. They have, however, a much greater grating vector $2 \pi / d$, meaning that their first evanescent order can be far beyond the near evanescent orders of a multi-mode resonant dielectric grating.

To blaze far evanescent orders, we combine a multimode resonant grating that can blaze near evanescent orders with a much shorter period single mode grating. The short period grating accepts a blazed near evanescent order as its dominant incident field and scatters it efficiently to far evanescent orders, broadening the spectrum and increasing resolving power commensurately. On the other hand, a small period single mode grating does not have the resonances necessary to enhance near or far evanescent orders, which is why a combination is required. This is shown in Fig. 7, where the addition of sub-period structuring to the bottom surface of the grating broadens the spectrum in transmission considerably. Similarly, Fig. 7 contrasts the narrow spectrum found on the upper unstructured surface (in reflection) and the much broader spectrum found on the lower structured surface in transmission.

To calculate these spectra and the fields they generate, the modal method must be generalized to deal with stacks of gratings with different periods. In this subsection we present a summary of our approach to this problem and the process of generalization.

One important aspect of the generalization is that the number of grating orders considered (which defines the size of the scattering matrices) needs to remain finite. For this reason, the ratio of the periods of the gratings has to be an integer ratio, ideally a low order one, so that the stacked structures remain periodic. This period $(D)$, which corresponds to the lowest common multiple of the constituent periods, becomes the period that defines the 


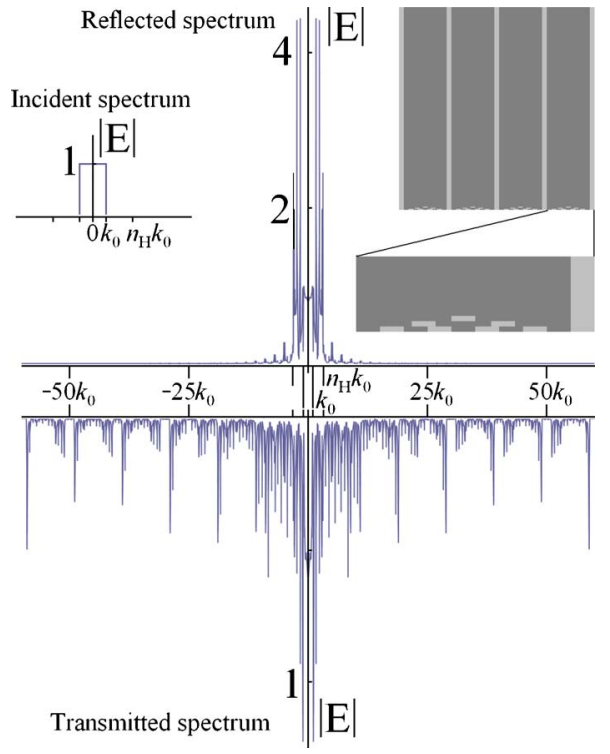

Fig. 7. (Color online) Absolute value of the incident, reflected, and transmitted spectrum from the $\mathrm{Si} / \mathrm{SiO}_{2}$ grating shown at the right. The long period grating has period $d=0.738 \mu \mathrm{m}$, thickness $t_{g}=3.08 \mu \mathrm{m}$, and duty cycle of $10 \%$. Each scattering grating layer has thickness $t_{s}=0.0148 \mu \mathrm{m}$.

grating orders the system operates with, as in Eq. (1). In general the constituent periods $d_{i}$ are less than the overall period $D$, so the natural orders of a grating with period $d_{i}$ are a factor of $D / d_{i}$ less dense than what is required to fully describe the system. This limitation is circumvented by calculating scattering matrices for the particular and artificial (and often evanescent) incident angles necessary to fill in the gaps, creating a set of orders of the required density. Each scattering matrix calculated for a given incident angle is a dense matrix describing interactions between orders congruent (modulo $D / d$ ), while all other orders remain independent. These dense matrices $\left(\left\{\mathbf{J}_{b}^{\prime}\right\}\right)$ are combined to form one larger matrix (J) which is valid for a single grating with period $d$, for a denser set of orders consistent with a structure of period $D$. In essence this amounts to a shortcut in calculating $\mathbf{J}$ since we can ignore orders that are not congruent (modulo $D / d$ ). This is achieved by a consistent reordering of $\alpha_{p}$ and $\beta_{n}$ originally calculated for each separate incident angle. This process is shown diagrammatically in Fig. 8. In this diagram, four change of basis matrices $\mathbf{J}^{\prime}$ calculated for a grating with period $D / 4$ are interleaved to form one larger matrix $\mathbf{J}$ which is valid for a set of orders four times as dense as would be necessary for a single grating with such a period.

A Fresnel scattering matrix for a stack of gratings is generated by combining the interleaved matrices for each individual grating according to a recursive formula analogous to the original modal method as outlined in Appendix C [Eq. (4a)]. Some care must be taken as, in general, a stacked system lacks up-down symmetry as shown in Fig. 9.

We next derive mathematically valid recurrence formulas for up-down asymmetric grating stacks. Each new grating addition brings three additional parameters: the spacing between the gratings $h$ (which we always set to

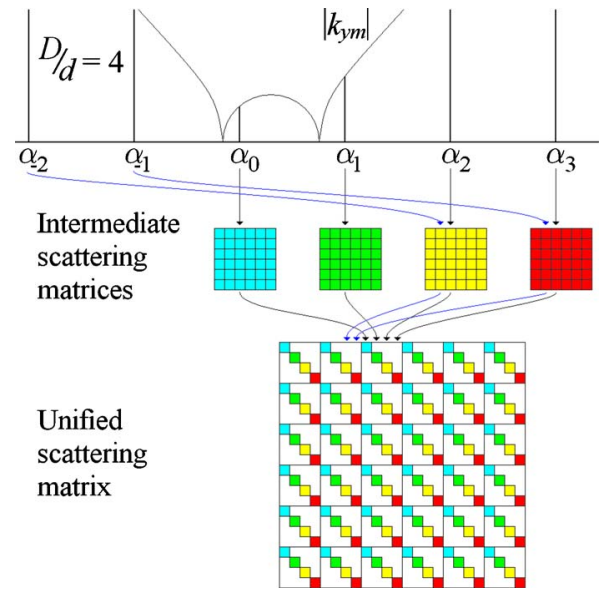

Fig. 8. (Color online) Diagram showing the interleaving technique used to construct scattering matrices for denser sets of orders (or a larger period) than the short period gratings naturally produce. The upper curve represents $\left|\chi_{m}\right|=\left|k_{y m}\right|$; propagating orders exist within the semicircle only.

zero), the thickness of the new grating $t_{s}$, and the horizontal offset between the phase origins of the topmost and bottommost gratings $x_{0}$. In this formulation new gratings are recursively added from below. As such, grating $s$ is updown symmetric but offset and separated, and grating $n$, which represents the current aggregate, is in general asymmetric. In this formulation, $\mathbf{T}_{i}$ and $\mathbf{R}_{i}$ refer to scattering matrices for downward energy propagation, while $\mathbf{T}_{i}^{\prime}$ and $\mathbf{R}_{i}^{\prime}$ refer to scattering matrices for upward energy propagation. For stacks of gratings lacking up-down symmetry they are not equivalent, so primes are retained in all the following formulas for full generality. Lastly, references to $\mathbf{T}$ and $\mathbf{R}$ in this section refer to the single grating scattering matrices derived in Appendix $\mathrm{C}$ with no offset, spacing, or anisotropy, but calculated with reference to the new denser set of plane-wave orders discussed in the previous paragraph.

As explained in Appendix B, we proceed by writing equations linking the amplitudes of the incident field $\delta$, the reflected field $r$, transmitted field $t$, and inter-grating plane-wave fields $\bar{c}^{ \pm}$. We distinguish $c^{ \pm}$, which are mode amplitudes, from $\bar{c}^{ \pm}$, which are plane-wave field amplitudes. The \pm sign distinguishes between upward and downward propagating fields. As in Fig. 9, inter-grating complex field amplitudes are defined at the point of reflection. That is, $\bar{c}^{-}$is defined at the upper grating, and vice versa. We find that

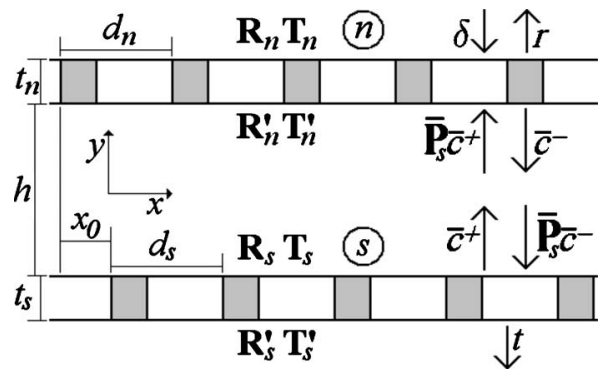

Fig. 9. Diagram of the configuration of fields and gratings used to derive the stacked grating recursion formula. 


$$
\begin{gathered}
r=\mathbf{R}_{n} \delta+\mathbf{T}_{n}^{\prime} \overline{\mathbf{P}}_{s} \bar{c}^{+}, \\
\bar{c}^{-}=\mathbf{T}_{n} \delta+\mathbf{R}_{n}^{\prime} \overline{\mathbf{P}}_{s} \bar{c}^{+}, \\
\bar{c}^{+}=\mathbf{R}_{s} \overline{\mathbf{P}}_{s} \bar{c}^{-}, \\
t=\mathbf{T}_{s} \overline{\mathbf{P}}_{s} \bar{c}^{-},
\end{gathered}
$$

where $n$ refers to the current aggregate and $s$ refers to the new addition as shown in Fig. 9. Here, $\overline{\mathbf{P}}_{s}=\exp (i \chi h)$ is the vertical propagation matrix since $\chi\left(=\operatorname{diag}\left\{\chi_{p}\right\}\right)$ is the matrix with $\chi_{p}$ on the diagonals. $\overline{\mathbf{P}}_{s}$ is not to be confused with $\mathbf{P}$, the analogous matrix used in the modal formulation of scattering matrices for single gratings. In our research, $h=0$ to maximize inter-order scattering efficiency, so $\overline{\mathbf{P}}_{s}=\mathbf{I}$ and can be left out.

Equations (5) can readily be solved to form $r=\mathbf{R}_{n+1} \delta$ and $t=\mathbf{T}_{n+1} \delta$, yielding matrices for the combined grating structure:

$$
\begin{gathered}
\mathbf{R}_{n+1}=\mathbf{R}_{n}+\mathbf{T}_{n}^{\prime} \overline{\mathbf{P}}_{s} \mathbf{R}_{s} \overline{\mathbf{P}}_{s}\left(\mathbf{I}-\mathbf{R}_{n}^{\prime} \overline{\mathbf{P}}_{s} \mathbf{R}_{s} \overline{\mathbf{P}}_{s}\right)^{-1} \mathbf{T}_{n}, \\
\mathbf{T}_{n+1}=\mathbf{T}_{s} \overline{\mathbf{P}}_{s}\left(\mathbf{I}-\mathbf{R}_{n}^{\prime} \overline{\mathbf{P}}_{s} \mathbf{R}_{s} \overline{\mathbf{P}}_{s}\right)^{-1} \mathbf{T}_{n} .
\end{gathered}
$$

Since we included primes in Eq. (6) for full generality, a simple symmetry argument can be used to derive $\mathbf{R}_{n+1}^{\prime}$ and $\mathbf{T}_{n+1}^{\prime}$, the upward energy propagating equivalents of $\mathbf{R}_{n+1}$ and $\mathbf{T}_{n+1}$, simply by inverting the use of primes (') and swapping $n$ and $s$ :

$$
\begin{gathered}
\mathbf{R}_{n+1}^{\prime}=\mathbf{R}_{s}^{\prime}+\mathbf{T}_{s} \overline{\mathbf{P}}_{s} \mathbf{R}_{n}^{\prime} \overline{\mathbf{P}}_{s}\left(\mathbf{I}-\mathbf{R}_{s} \overline{\mathbf{P}}_{s} \mathbf{R}_{n}^{\prime} \overline{\mathbf{P}}_{s}\right)^{-1} \mathbf{T}_{s}^{\prime}, \\
\mathbf{T}_{n+1}^{\prime}=\mathbf{T}_{n}^{\prime} \overline{\mathbf{P}}_{s}\left(\mathbf{I}-\mathbf{R}_{s} \overline{\mathbf{P}}_{s} \mathbf{R}_{n}^{\prime} \overline{\mathbf{P}}_{s}\right)^{-1} \mathbf{T}_{s}^{\prime} .
\end{gathered}
$$

For the purpose of recursion, $\mathbf{R}_{n}$ and $\mathbf{T}_{n}$ are initialized $(n=1)$ according to

$$
\begin{aligned}
& \mathbf{R}_{1} \equiv \mathbf{R}_{1}^{\prime}=\mathbf{R}, \\
& \mathbf{T}_{1} \equiv \mathbf{T}_{1}^{\prime}=\mathbf{T},
\end{aligned}
$$

where $\mathbf{T}$ and $\mathbf{R}$ refer to the single grating scattering matrices derived in Appendix $\mathrm{C}$ with no offset, spacing, or anisotropy, but calculated for the complete set of planewave orders as previously explained.

Lastly, although $\mathbf{R}_{s}=\mathbf{R}_{s}^{\prime}$ and $\mathbf{T}_{s}=\mathbf{T}_{s}^{\prime}, \mathbf{R}_{s} \neq \mathbf{R}$ and $\mathbf{T}_{s} \neq \mathbf{T}$, as $\mathbf{R}_{s}$ and $\mathbf{T}_{s}$ represent matrices horizontally translated by some offset $x_{0}$. This offset is, however, readily taken into account by shifting the phase origin using

$$
\begin{aligned}
\mathbf{R}_{s} & =\mathbf{Q}^{-1} \mathbf{R Q}, \\
\mathbf{T}_{s} & =\mathbf{Q}^{-1} \mathbf{T} \mathbf{Q},
\end{aligned}
$$

where $\mathbf{Q}=\exp \left(i \boldsymbol{\alpha} x_{0}\right) . \boldsymbol{\alpha}\left(=\operatorname{diag}\left\{\alpha_{p}\right\}\right)$ is the matrix with $\alpha_{p}$ on the diagonals.

As computational speed at least partially depends on the speed with which matrices can be inverted, the smaller the matrices for a given spectral width, the better. For this reason we choose to use gratings with period ratios of $1: P(P$ integer $)$ and to limit the number of gratings in a given stack. The method, however, is general. Once completed, scattering matrices can be calculated as before, generating spectra and fields in any region of interest, as demonstrated throughout this paper.

The efficiency with which the small period grating transmits the near evanescent order enhancement outward is now the governing factor for the extension of resolving power. Important factors include the period ratio, thickness, duty cycle, alignment (necessary to preserve left-right symmetry), and refractive index contrast. Stacking gratings with different periods is the key innovation presented in this paper, as it provides an efficient way of calculating the coupling of information and energy between the previously invisible and spatially localized far evanescent fields and propagating fields which can be imaged in the far field.

\section{Fourier Model}

The generalization of the modal method for stacking gratings with a wide range of possible geometries is universally applicable. We use a Fourier model to develop some guiding principles of grating design, based on our goal of synthesizing an isolated, steerable, and narrow spot.

The Fourier model is motivated by the observation that gratings spread the horizontal component of the spatial frequency in accordance with Eq. (1). This is at the core of the issue surrounding the broad spectrum necessary to generate sub-wavelength spots and thus attain resolving power beyond the Rayleigh limit. To illustrate the general point, the spatial Fourier transform of a narrow Gaussian spot is a Gaussian spectrum, where the widths are inversely proportional.

In accordance with this central idea, we review the Abbe criterion on resolution [24]. A propagating incident field is fundamentally limited in its horizontal resolving power by the width of spectrum available in propagating orders. Even with a (hypothetical) lens system permitting $2 \pi \mathrm{rad}$ of incident field (in two dimensions), the horizontal components of the spectrum are confined such that $\left|k_{x}\right|<k_{0}=2 \pi / \lambda$. This is the limiting factor and the reason why conventional imaging or spot synthesis is unable to image features smaller than $\lambda / 2$. To generate a narrower spot requires high frequency evanescent waves with spectral field components beyond $k_{0}$.

As discussed at the beginning of Subsection 2.C, a single grating combining a Fabry-Perot resonance and TIR can broaden the spectrum by a factor of $n_{H}$, with a corresponding minimum spot width of $\lambda / 2 n_{H}$. To go beyond that, a stack of gratings with different periods is needed; such a system scatters energy to yet more remote orders with reasonable efficiency, effectively broadening the envelope and thus narrowing the spot by about the same factor.

It is one thing to generate very narrow spots-there are several known ways to do this, such as using tiny holes in thin metal sheet [25], which is a very energy inefficient process. The advantage of the grating approach is that the spots are generated not through some masking process but via spectral synthesis, in an efficient way. The spots are not locked in location to isolated surface features and can potentially be generated in a continuous range of locations across the surface. 
Prima facie, it is not obvious that spots generated using stacked gratings are in any way steerable. A stacked grating typically produces a comb-like spectrum, and as Fig. 10 illustrates, the Fourier transform gives rise to a strong correspondence between the shapes of the spatial and spectral profiles. That is, a comb-like spectrum produces a comb-like field profile with a peaked envelope. Even if the envelope can be shaped to favor only one peak, the positions of the peaks are both fixed and widely separated.

This apparent problem can be solved. In more detail, Fig. 10 shows that the width of the spectrum is inversely proportional to the width of the spot, while the width of each peak within the spectrum corresponds to the width of the spatial envelope of the field. The spectral peak spacing is inversely proportional to spatial peak spacing. With this in mind, reverse engineering of the spectrum may be utilized. In order to produce an isolated spot, the spatial envelope must be narrower than the spot spacing. In particular, narrow spots may only be generated at certain discrete positions under a movable envelope. In a two grating stack with a period ratio of $1: P$, these positions are equally spaced with $P$ in each period. One way of circumventing this problem is to use a large valued $P$, such that the spot spacing $D / P$ is smaller than the minimum spot size, and then attempt to excite such closely spaced spots independently. Unfortunately this method requires a large period ratio $P$ which is less efficient at scattering to far evanescent orders, and in general the spatial envelope remains too wide to excite narrow spots independently. An alternative method is to use a stack of several gratings with coprime $P$ values, such that there is an abundance of potential spot locations despite the relatively low values of $P$.

Additionally, the production of a steerable spot requires a field with isolated and independently excitable narrow spots. This requires a broad spectrum, which provides clues for grating design. A 1:P grating stack produces a spectrum with peak spacing proportional to $P$. To avoid spaces developing in the spectrum (which would have the effect of creating large gaps where spots could not be generated), several thin gratings of different periods must be combined. Period ratios of 1:2:3:5 ensure that every space out to the next consecutive prime number (7) is at least partially filled as shown in Fig. 11.

With a lowest common multiple of 30 , the 1:2:3:5 grating is certainly calculable. In comparison, $1: 2: 3: 5: 7$ $\rightarrow 210$ is $>7^{3}$ times the effort [matrix inversion is $\mathcal{O}\left(n^{3}\right)$ ] for a $40 \%$ increase in resolution. A 1:2:3:5 grating stack is

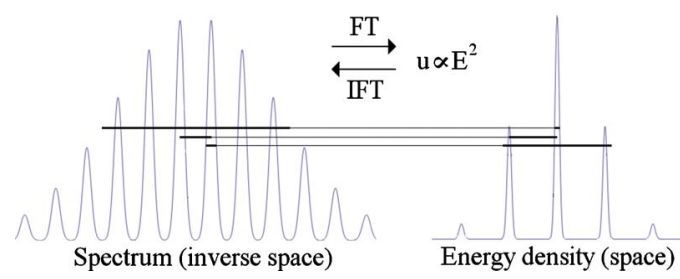

Fig. 10. (Color online) Correspondences between spectral and spatial spot characteristics due to the Fourier transform. The width of the spectrum is inversely proportional to the width of the spot, while the width of each peak within the spectrum corresponds to the width of the spatial envelope. Spectral peak spacing is inversely proportional to spatial peak spacing.

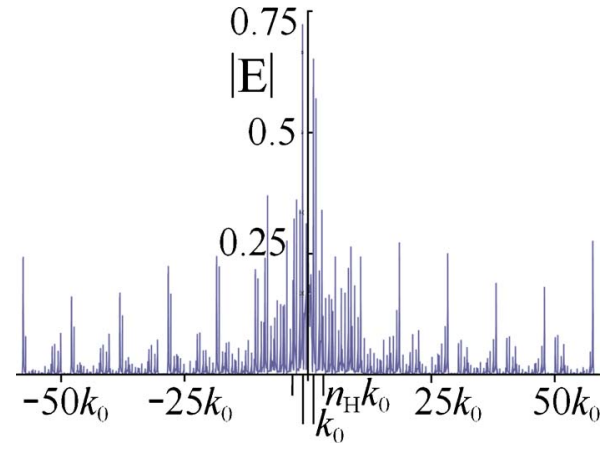

Fig. 11. (Color online) Amplitude of the spectrum of the leftmost spot in Fig. 13.

capable of producing an effectively continuous distribution of spots as small as $\lambda / 20$ as shown in Section 4 .

\section{E. General Design Considerations}

In the next section, we describe an optimization algorithm for stacked dielectric lamellar gratings and the results it generated. However, the construction of the algorithm was preceded by many numerical experiments, which gave rise to physical insights incorporated into the algorithm, and which underlay its results. Here, we discuss a number of these.

Our design is based around lamellar gratings, which feature sharp corners in the silicon/silica interface. Such sharp corners require large numbers of modes to be present within the gratings and large numbers of plane waves to be present in air. This means that in air and the gratings we have dense spectra of evanescent waves, which are available for coupling at a wide range of incidence angles. This suggests that sharp profile corners aid in the construction of sub-wavelength steerable spots. However, the occurrence of field concentrations at the corners means that absorption, if present in the grating material, would be enhanced there [26], favoring designs based on dielectrics. Evanescent mode enhancement via sharp corners also works better in the presence of strong contrast of dielectric permittivity, favoring the use of a high index material like silicon [27].

The next major design choice is that of the grating periods incorporated in the structure. We have already described the kinematic arguments based around the grating equation which favor a stack of four gratings with periods bearing prime ratios, and with the smallest period being in the range accessible with current lithographic techniques (just below $0.20 \mu \mathrm{m}$ ).

The thickness $t_{g}$ of the primary grating is an important parameter. This is not chosen in order to make a single mode resonate with a high quality factor. Rather, our requirement is to have available a rich spectrum of resonances with low to moderate $Q$ 's (around 5) so that one can move smoothly from one to the next as the angle of incidence is varied. The best appropriate range of $t_{g}$ is found by examining the variation of the mode resonance spectrum with thickness and choosing the range (around four times the period for the indices used here) where a rich spectrum of appropriately overlapping resonances is generated. Given the choice of the primary grating, the finer gratings are built into the design: their thickness 
must be sufficient so they can scatter energy efficiently and comprehensively into far evanescent orders, thus generating the sub-wavelength spots. It should be noted that evanescent order resonances can be quite strong with small values of $t_{g}$ in a silicon/silica grating. (The lamellar grating modal method is well adapted for this part of the optimization process, since the bulk of the calculation time required to solve a diffraction problem for it is independent of the grating thickness.)

The final result of the optimization is a stack of four dielectric gratings, generating a full spectrum of moderate $Q$ resonances. As the angle of incidence varies, the stack moves smoothly from one resonance to the next, generating a steerable sub-wavelength spot. As a consequence of the overlapping nature of these resonances, the fields within the grating stack are quite complicated in form, with large amplitudes of highly evanescent grating orders.

\section{OPTIMIZATION BY MCMC}

In the system designed in the way just described, a stacked grating structure has many resonances accessible by incident fields from different angles. As seen in Fig. 5, there are a large number (between 30 and 60) of different angles, each of which represents two scalar variables (amplitude and phase). Each blazing angle gives rise to a field which, while quasiperiodic, is anything but spot-like. The synthesis of customized fields on the grating surface through the adjustment of up to 120 parameters requires a robust and effective optimization process. In this research, we used a MCMC to locate quickly and efficiently a local maximum of a peak-like merit function through this large parameter space. A MCMC works by taking a directed random walk through parameter space in search of maxima (or minima) of an arbitrary merit function [28]. Traditionally associated with efficient assessment of posterior probability distributions in Bayesian statistics, a MCMC may be readily employed as a simple and versatile optimizer. It differs from many calculus-based optimizers in its extensive use of random numbers.

A first approximation to the incident field is needed to initialize the optimizing algorithm. The approach we used was to calculate a least-squares solution of the incident field. The transmitted spectrum was matched as closely as possible to the spectrum of an ideal spot (in the correct position) obtained using a Fourier transform. While adequate as a first approximation, the results of the leastsquares approach serve only as a starting point for the optimizer. For the purpose of generating a narrow spot our merit function (c) simply assessed the ratio of energy $\left(\sim|E|^{2}\right)$ across the narrow region of desirable spot location to a wider background region of width $2 D \approx \lambda$.

At each step of the optimization process the MCMC augments a given incident field by a small random increment and calculates the new position's merit $c^{\star}$. The merits of the two incident fields are compared by taking their quotient $\left(c^{\star} / c\right)$, and a forward step is taken if the merit of the new position is greater than the previous incident field. To prevent the chain from becoming trapped in small local maxima, the chain is also allowed to step downhill with a certain probability proportional to the ra- tio of the merits. For a high dimension parameter space, an acceptance rate of $24 \%$ is heuristically optimal. Our chain monitors the acceptance rate continuously (by recording the acceptance rate over the previous ten steps) and adjusts various parameters to ensure the highest efficiency. One such parameter is the merit ratio "temperature" $(T)$. By taking the merit ratio to the power $T$ $\left[\left(c^{\star} / c\right)^{T}\right]$, with $T>1$, the contrast in the merit function between peaks and valleys is increased. The temperature $T$ is also used to regulate the step size and ensure that the optimization proceeds efficiently. This is seen in Fig. 12, where a steady increase in the merit function (right) is accompanied by a decrease in the temperature (left) to a low background level. The oscillatory behavior of the temperature near an optimized peak actually increases the volatility of the chain and helps to maximize the efficiency. On average, our MCMC was able to find a satisfactory localized spot in under 100 steps, increasing the phase aligned initial field's merit by a factor of about 5 . This increase corresponds to a substantial narrowing of the spot and the minimization of background noise. The optimization process is repeated for any given position where a spot is required.

To generate a continuous distribution of spots as seen in Fig. 13, a separate optimization is run for each spot. A typical temperature and merit variation across many parallel chains is shown in Fig. 12. The temperature is initialized at a high value, and then falls as the chain is optimized. Beyond the optimal point, the temperature often becomes quite volatile as the chain hunts for further optimal solutions. Meanwhile the merit rises and peaks. In most of the chains, this occurs very quickly; however a few positions along the unit cell are particularly well suited to very low background noise, giving rise to a better merit function. As the merit function searches for a region much narrower than any given spot, the apparently low values achieved $(\sim 0.3)$ are not very indicative of the final spot quality. All that is necessary for a good merit function is positive definite behavior in the direction of optimization.

An advantage of using an MCMC approach for optimization as opposed to steepest descent/ascent methods is that an MCMC does not require a derivative to be taken. As the electric field is composed of a superposition of thousands of independent orders, this represents a substantial saving in computational complexity. This advantage does not apply, however, for optimizing electric field profiles under TM polarization as the curl of the field must be taken, slowing the process considerably. For convenience we restrict the optimization of spots in this pa-

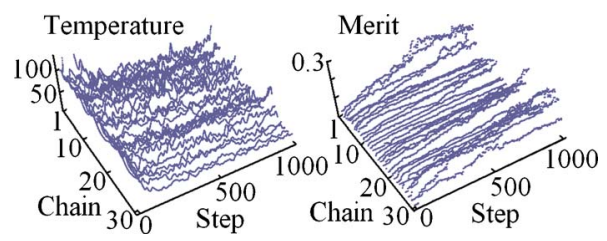

Fig. 12. (Color online) Details of 30 parallel optimizing processes over 1000 steps. The left figure shows the temperature of each chain as it converges on a local maximum. The right figure shows the progress of the merit function as it trends from a low initial position to an optimized position. 


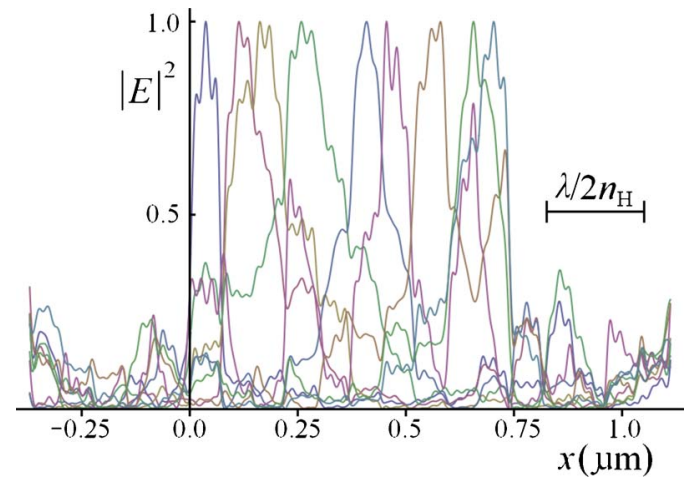

Fig. 13. (Color online) Energy density of a series of optimized spots across a single period $d=0.738 \mu \mathrm{m}$ below the grating shown in Fig. 7.

per to the TE polarization; however the blazing of TE or TM evanescent orders is analogous and equally valid in either polarization, for dielectric structures.

\section{RESULTS AND DISCUSSION}

The optimization process attempts to build closely spaced peaks across the surface of a period of the grating. Its success depends on the heuristic suitability of the grating for continuous peak generation and the efficiency of the optimizer. Ultimately, as seen in Fig. 12, the optimizer reaches a ceiling value for a given location, indicating that the spot width has reached the limit imposed by the spectral width and the independence of the blazed orders. In this section, we discuss the results from one optimized grating and examine the robustness of the optimized peaks.

We use the wavelength $(1.55 \mu \mathrm{m})$ and the corresponding refractive index of silicon $\left(n_{\mathrm{Si}}=3.41\right)$ and increase the number of grating orders used in the calculation to 60 per incident angle, effectively doubling the resolution of the calculation in comparison to our previous paper. As shown in Fig. 13, this increase in the calculation resolution demonstrates a substantial sub-structure present in optimized spots, but does not produce spots that are narrower than the sub-wavelength spots shown in our previous report [10]. The width of the spots is limited by the width of the blazed spectrum, which is limited in turn by the efficiency of far evanescent order blazing. For this reason, there is limited utility in further increasing the number of orders, and thus the resolution, beyond 60 to 90 or 120 .

We performed a robustness analysis on the results by blurring (by adding uniform random noise) the incident field, removing incident channels, or truncating the most extreme evanescent orders in the calculation. In Fig. 14, the amplitude and phase of the optimized incident fields shown in Fig. 13 have been detuned by the addition of a uniform random complex component within a bound of 10\%. As shown in Fig. 14, the peak behavior of each spot is preserved, while amplitude varies and background noise increases slightly. This exercise in random perturbation of the incident field demonstrates the robust nature of evanescently generated spots. This is in contrast to super-oscillations [29], which are low amplitude subwavelength peaks generated by careful interference of

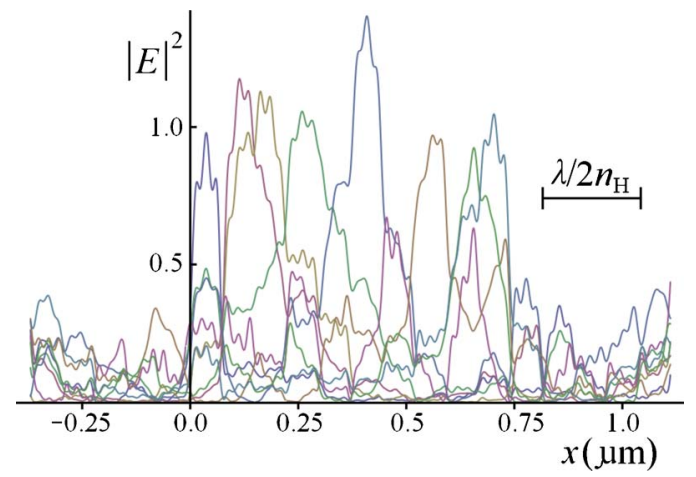

Fig. 14. (Color online) Diagram showing a set of spots where the previously normalized optimized incident fields in Fig. 13 have been subjected to $10 \%$ random variation or blurring.

propagating waves, which do not demonstrate any degree of robustness to incident field perturbations. Furthermore we found that the spot-like character of optimized fields is also robust to the complete removal of an incident channel, which permits order-by-order optimization in the real device similar to that employed by Vellekoop and Mosk [30]. Additionally, the spot was robust with respect to the truncation of the generated spectrum. In this instance, we removed all spectral components corresponding to the outermost elements of the constituent scattering matrices in an effort to quantify the role of the truncation error when generating spot-like fields. As may be expected, the removal of field elements corresponding to relatively lowamplitude features around five times narrower than the (already narrow) spots had little overall effect.

We also considered a geometry in which spots were optimized subject to a $2 \pi$ field, incident from both above and below. This could be practically achieved with a mirror a short distance above the spots to be optimized or through the use of a second incident wave input system, as assumed in this particular calculation. The rationale behind this was to mimic the omnidirectional nature of radiation from a delta function source at a particular point on the grating surface, under time reversal reciprocity. Despite the substantial increase in independence achieved through a doubling of the number of input channels, we found that the spot width is fundamentally limited by the far evanescent order scattering efficiency of the grating rather than by the numerical aperture.

We investigated the extent to which resonant behavior and evanescent order blazing were affected by a supporting grating substrate. This was achieved by adding another layer of a grating with identical refractive indices in both rectangles to the top of the stack. In our case we used a silica superstrate $\left(n_{\mathrm{SiO}_{2}}=1.46\right)$ that was $1 \mathrm{~mm}$ thick. Figure 15 shows the incident field [Fig. 15(a)], the spectrum in transmission of a multi-layered $\mathrm{Si} / \mathrm{SiO}_{2}$ grating suspended in vacuum [Fig. 15(b)], the same spectrum of the same grating with a superstrate [Fig. 15(c)], and the geometry of the grating [Fig. 15(d)]. While the exact position of the resonances is modified, the resonant character, and blazing of the evanescent field, is still present. This shows that grating substrates or superstrates do not substantially affect the character of both near and far evanescent order blazing. 


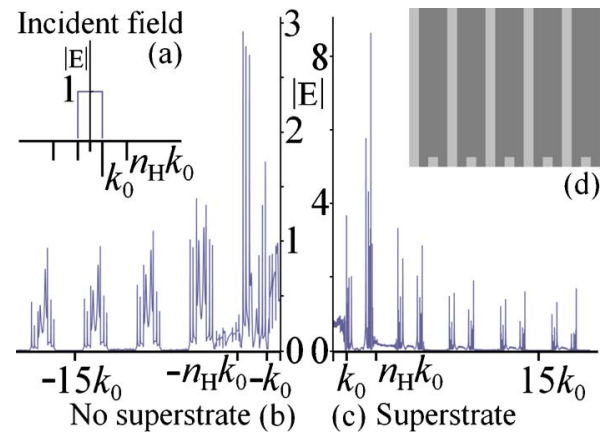

Fig. 15. (Color online) (a) Spectrum of incident field; unit amplitude in every incident angle calculated. (b) Spectrum in transmission of grating shown in (d). (c) Spectrum in transmission of grating shown in (d) with a $1 \mathrm{~mm}$ superstrate above the grating. (d) Diagram of a stacked grating thickness $t_{g}=2.87 \mu \mathrm{m}$, period $d=0.738 \mu \mathrm{m}$, with a duty cycle of $25 \%$. Sub-period ratio (1:2) and scattering grating thickness $t_{s}=0.148 \mu \mathrm{m}$. The data in this figure alone were calculated with $n_{H}=3.61$.

We also present an optimized series of spots attained with a bare minimum of incident channels, examining the trade-off between less order independence and operational complexity. Figure 16 shows spots optimized with only 18 incident angles (instead of 54), corresponding to only the strongest resonances visible in the propagating order as shown in Fig. 5. The reduction in incident channels by a factor of 3 slightly affects the sharpness of optimized spots, but at a substantial reduction in the complexity of the incident field. Additionally, the optimizer failed to produce spots at certain locations, leaving some gaps across which the narrow spot must jump. Just one well chosen incident field is adequate to excite the strong evanescent waves necessary for FFODT; somewhat more are needed to synthesize particular fields on the grating surface.

We believe that a FFODT system exploiting the principles of evanescent order blazing could be readily built using a spatial light modulator (SLM) and wide-angle objective lens system employed by Vellekoop and Mosk, when they achieved universal optimal transmission of light through disordered materials [30]. Under the resolution used in our calculation (400 incident angles between $-90^{\circ}$ and $90^{\circ}$ ), each incident angle is separated by about $0.45^{\circ}$. To achieve accurate excitation of resonances, most of which are of the order of this angular width, an incident plane wave should have at least twice this reso-



Fig. 16. (Color online) Diagram showing energy density for a set of spots optimized with only 18 (instead of 54) incident angles. lution. A state-of-the-art SLM has a resolution similar to a high end LCD monitor, with the number of pixels of the order $2000 \times 2000$. A SLM used to modulate both amplitude and phase needs four $(2 \times 2)$ pixels for each element of phase front [31], which means that there are 1000 "channels" available (in two dimensions) for exploitation along any given axis. This compares favorably to the 400 incident angles used in the calculation, even when losses occur due to a mirrors-and-lenses geometric transformation from a planar incident field to a $180^{\circ}$ circular incident field.

In this system, the subject would be carefully placed on a specialized microscope slide with a resonant grating layer on one surface. While gratings such as those shown in Fig. 7 are non-trivial to construct, several precedents exist in lithography for generating gratings with these features, either through layered deposition and doping or directly through doping with different masks and dopants with different energies, each penetrating to its own characteristic depth. Additionally, the far evanescent order blazing technique works for any two gratings with integer period ratios provided one of them displays resonant behavior. The governing factor is the spectral profile generated by a given combination. Using the heuristics described using the Fourier model and exploiting left-right symmetry for computational efficiency, we arrived at a particular grating design; however, it is by no means the only possible geometry.

\section{CONCLUSION}

Sub-wavelength optical microscopy is the next frontier in in vivo biological studies and nanolithography, among other topics. In Section 2, we outlined the modal method, explained the generalization to stacks of gratings with different periods, and explained the phenomenology of modal resonances in dielectric gratings, as well as the spectral effects of grating stacks with a Fourier model. In Section 3 we explained our high dimensional optimization process through a Monte Carlo Markov chain (MCMC), and in Section 4 we demonstrated the robust and substantially sub-wavelength nature of our optimized spots. Through our research we have shown that a stack of dielectric gratings with different periods supported by a glass substrate is a viable approach to the spectral synthesis necessary to efficiently transport sub-wavelength information into the far field, and without the introduction of fluorescent scatterers or physical probes. While our discussion has been centered at lamellar gratings, we stress that our approach could be used for other grating profiles. It is a non-trivial question worthy of further investigation as to whether metallic gratings could outperform dielectric gratings.

\section{APPENDIX A: FIELD DECOMPOSITION IN FREE SPACE AND LAMELLAR GRATING}

The method begins with the Helmholtz equation, which is to be solved in two dimensions within one period of the grating as shown in Fig. 2. In this configuration there are two separable polarizations: $E_{z}$ and $H_{z}$. While the modal 
method is equally valid for either polarization, and includes both in full generality, from here on we will deal exclusively with the $E_{z}$ polarization, as explained in Section 3. That is,

$$
\mathbf{E}=\sum_{n} a_{n} f_{n}(x) e^{i \beta_{n} y} \mathbf{z}
$$

for some orthonormal set of $x$-variation functions $f_{n}(x)$, each with amplitude $a_{n}$, and a $y$ spatial frequency $k_{n}$. In this case $f_{n}(x)$ is composed of a linear superposition of two orthonormal solutions $[\theta(x)$ and $\psi(x)]$ to the Helmholtz equation in the grating as outlined below.
As the Helmholtz equation is a second order differential equation, there are two independent solutions. For simplicity, we choose orthonormal solutions $\theta(x)$ and $\psi(x)$, satisfying

$$
\begin{aligned}
& \theta(0)=\frac{\partial}{\partial x} \psi(0) \equiv \psi^{\prime}(0)=1, \\
& \psi(0)=\frac{\partial}{\partial x} \theta(0) \equiv \theta^{\prime}(0)=0 .
\end{aligned}
$$

Under these conditions, there is a sine- and cosine-like pair of independent solutions (for $E_{z}$ polarization) given by

$$
\begin{gathered}
\theta(x)= \begin{cases}\cos \gamma_{1} x & \text { if } 0<x<c_{1} \\
\cos \gamma_{1} c_{1} \cos \gamma_{2}\left(x-c_{1}\right)-\frac{\gamma_{1}}{\gamma_{2}} \sin \gamma_{1} c_{1} \sin \gamma_{2}\left(x-c_{1}\right) & \text { if } c_{1}<x<d,\end{cases} \\
\psi(x)= \begin{cases}\frac{1}{\gamma_{1}} \sin \gamma_{1} x & \text { if } 0<x<c_{1} \\
\frac{1}{\gamma_{1}} \sin \gamma_{1} c_{1} \cos \gamma_{2}\left(x-c_{1}\right)+\frac{1}{\gamma_{2}} \cos \gamma_{1} c_{1} \sin \gamma_{2}\left(x-c_{1}\right) & \text { if } c_{1}<x<d .\end{cases}
\end{gathered}
$$

Here $\gamma_{1,2}=\sqrt{k_{0}^{2} \epsilon_{1,2}-\beta^{2}}$, while $\epsilon$ represents the permittivity of each region. Then under this modal decomposition of the field in the grating, $\beta$ is the propagation constant of a given mode perpendicular to the interface, $\gamma_{1}$ is the transverse wave number parallel to interface in region 1 , and $\gamma_{2}$ is the corresponding transverse wave number in region 2. As written, $\theta(x)$ and $\psi(x)$ describe a continuous spectrum of grating modes indexed by the vertical propagation parameter $\beta$. The boundary condition of quasiperiodicity under a given incident angle $\phi_{0}$ breaks the continuous spectrum into a countably infinite subset of modes which are actually permitted in the structure under a particular incident plane-wave field.

Defining $A$ and $B$ to be the respective amplitudes of the $\theta(x)$ and $\psi(x)$ modes and the Bloch factor across one period $\tau=\exp \left(i k_{0} d \sin \phi_{0}\right)$ (see Fig. 2), the quasiperiodicity condition $E(x+d)=\tau E(x), E^{\prime}(x+d)=\tau E^{\prime}(x)$ can be written as follows:

$$
\begin{aligned}
\left(\begin{array}{cc}
\theta(d) & \psi(d) \\
\theta^{\prime}(d) & \psi^{\prime}(d)
\end{array}\right)\left(\begin{array}{l}
A \\
B
\end{array}\right) & =\tau\left(\begin{array}{cc}
\theta(0) & \psi(0) \\
\theta^{\prime}(0) & \psi^{\prime}(0)
\end{array}\right)\left(\begin{array}{l}
A \\
B
\end{array}\right)=\tau\left(\begin{array}{ll}
1 & 0 \\
0 & 1
\end{array}\right)\left(\begin{array}{l}
A \\
B
\end{array}\right) \\
& =\tau\left(\begin{array}{l}
A \\
B
\end{array}\right)
\end{aligned}
$$

or

$$
\left(\begin{array}{cc}
\theta(d)-\tau & \psi(d) \\
\theta^{\prime}(d) & \psi^{\prime}(d)-\tau
\end{array}\right)\left(\begin{array}{l}
A \\
B
\end{array}\right)=0,
$$

which is an eigenvalue problem. Taking the determinant we arrive at

$$
\theta(d) \psi^{\prime}(d)-\psi(d) \theta^{\prime}(d)-\left(\theta(d)+\psi^{\prime}(d)\right) \tau+\tau^{2}=0 .
$$

The first two terms form the Wronskian which, under our choice of $\theta(x)$ and $\psi(x)$, is always equal to 1 . Thus

$$
\theta(d)+\psi^{\prime}(d)=\frac{1+\tau^{2}}{\tau}=2 \cos \alpha_{0} d
$$

where $\alpha_{0}=k_{0} \sin \phi_{0}$. We rewrite this in terms of sines and cosines and arrive at the Kronig-Penney equation for $\beta$ (via the terms $\gamma_{1}$ and $\gamma_{2}$ ):

$$
\begin{aligned}
& 2 \cos \gamma_{1} c_{1} \cos \gamma_{2}\left(d-c_{1}\right)-\left(\frac{\gamma_{1}}{\gamma_{2}}+\frac{\gamma_{2}}{\gamma_{1}}\right) \sin \gamma_{1} c_{1} \sin \gamma_{2}\left(d-c_{1}\right) \\
& =2 \cos \alpha_{0} d .
\end{aligned}
$$

Solutions of Eq. (A8) give the grating modes $\theta_{n}$ and $\phi_{n}$. Figure 17 shows how modal properties vary as functions of the angle of incidence. Plotting as a function of $\beta^{2}$ is an effective method of restricting propagating and evanescent solutions to a $2 \mathrm{D}$ graph provided both media are dielectric. The curved line, corresponding to the left side of the Kronig-Penney equation, varies with the square of the vertical propagation constant $\left(\beta^{2}\right)$, while the intermediate horizontal line, corresponding to the right side of the equation, varies with the incident angle $\phi_{0}$ by moving vertically between the two outer horizontal lines $( \pm 2)$. Where the lines intersect gives the solutions of the equation: modes that satisfy the quasiperiodic condition and exist within the structure under a given angle of incidence. Each subsequent mode, existing as a particular superposition of $\theta(x)$ and $\psi(x)$ solutions, exhibits either odd 


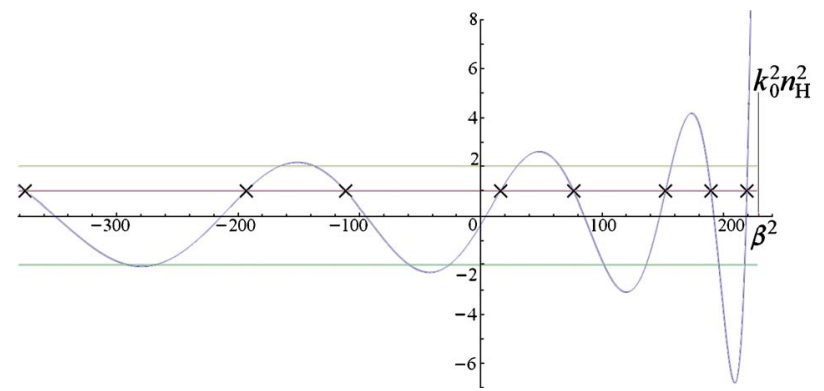

Fig. 17. (Color online) Plot of the left and right hand sides of the Kronig-Penney equation as a function of $\beta^{2}$. Points of equality or intersection are marked with crosses, corresponding to the modes that exist under a given angle of plane-wave incidence. Varying incident angle varies the height of the intermediate horizontal line between its two extrema.

or even symmetry and an integer number of oscillations within one period. Crucially, under certain conditions modes resemble each other closely enough to interact and produce avoided crossings as seen in Fig. 4. Only the modes that correspond to solutions on the positive side of the vertical axis in Fig. $4\left(\beta^{2}>0\right)$ are propagating within the structure; all others are evanescent.

At this point the horizontal dependence of the field has been expressed in terms of a discrete spectrum of grating modes, while the vertical variation (where needed) is accounted for by including a factor $e^{i \beta_{n} y}$. In this way the horizontal field variation on the boundary of the grating can be defined as a sum across all modes of both solutions of the Helmholtz equation,

$$
u_{n}(x)=A_{n} \theta_{n}(x)+B_{n} \psi_{n}(x),
$$

where $A_{n}$ and $B_{n}$ are the eigenvectors corresponding to Eq. (A5), and $n$ is the mode index, according to the conventional mode ordering $\beta_{i}^{2}>\beta_{j}^{2}$ for $i<j$.

The electric field at the dielectric-free space boundary may also be decomposed in terms of the plane-wave orders given by Eq. (1). The horizontal wave numbers $k_{x m}$ are relabeled as $\alpha_{p}=\alpha_{0}+2 \pi p / d$ and ordered directly by their indices $p$. The electric field is a sum of orthonormal components $e_{p}(x)$ given by

$$
e_{p}(x)=d^{-1 / 2} e^{i \alpha_{p} x}
$$

where $d$ is the grating period. Under this ordering, propagating waves correspond to the values of $|p|$ near zero and are found in the middle of the set.

With the solution of the Kronig-Penney equation, it is possible to describe the modes within the structure in a unified manner. In the limit $c_{1} / d \rightarrow 1$ (or zero) the modes tend asymptotically to plane waves in a homogeneous dielectric slab with permittivity $\epsilon_{1}$ (or $\epsilon_{2}$ ). In more general structures, however, they are characterized by the three interdependent propagation constants (which differ in each polarization) $\left(\gamma_{1}(\beta), \gamma_{2}(\beta), \beta\right)$, governing their horizontal behavior in the two media and the vertical direction as either evanescent or propagating as shown in Fig. 3 . Only propagating modes can transmit energy vertically through the grating, and thus these modes give observable manifestations of grating resonances. The strength of the resonances depends on the degree to which propa- gating grating modes couple to propagating plane-wave orders-the weaker the coupling, the stronger the resonance.

The resonances are of a Fabry-Perot nature, and thus the resonant condition depends on $\beta$, the distance between the upper and lower surfaces of the grating, and the phase shift acquired on reflection.

\section{APPENDIX B: FIELD MATCHING, CHANGES OF BASIS, AND FRESNEL SCATTERING MATRICES}

In this appendix, we derive the scattering matrices $\mathbf{R}_{12}$ and $\mathbf{T}_{12}$ for coupling at the interface from free space into grating modes and $\mathbf{R}_{21}$ and $\mathbf{T}_{21}$ for the reverse coupling. As constructed, $\left\{u_{n}(x)\right\}$ and $\left\{e_{p}(x)\right\}$ are an orthonormal basis, i.e.,

$$
\begin{gathered}
\int_{0}^{d} u_{n}(x) \bar{u}_{m}(x) \mathrm{d} x=\delta_{n m}, \\
\int_{0}^{d} e_{p}(x) \bar{e}_{q}(x) \mathrm{d} x=\delta_{p q} .
\end{gathered}
$$

At the core of the scattering matrix calculation is the (analytically soluble) mode-order overlap matrix $\mathbf{J}$ given by

$$
J_{p n}=\int_{0}^{d} \bar{e}_{p}(x) u_{n}(x) \mathrm{d} x=\int_{0}^{d} d^{-1 / 2} e^{-i \alpha_{p} x} u_{n}(x) \mathrm{d} x,
$$

where $\bar{e}_{p}$ is the complex conjugate of $e_{p}$ such that

$$
u_{n}(x)=\sum_{p} J_{p n} e_{p}(x) .
$$

The relation $u_{n}(x)=\Sigma_{p} J_{p n} e_{p}(x)$ is now generalized in terms of an arbitrary input and output field. The complete $2 \mathrm{D}$ plane-wave field in reflection (in terms of $E_{z}$ ) is defined by

$$
E_{\mathrm{pw}}(x, y)=\sum_{p} \chi_{p}^{-1 / 2}\left(r_{p} e^{i \chi_{p} y}+\delta_{p} e^{-i \chi_{p} y}\right) e_{p}(x),
$$

where $r$ represents the plane-wave order amplitudes of the reflected field, and $\delta$ represents the corresponding amplitudes of the incident field as shown in Fig. 18. The $\chi_{p}^{-1 / 2}$ term is a normalizing factor which is included to scale geometric factors out of energy flux calculations. In all cases the complex root is given by the principal part.



Fig. 18. Diagram showing the relative directions and positions of orthonormal mode/order amplitude vectors. 
Consider a semi-infinite grating region. The corresponding complete downward oriented modal field in the grating is written as

$$
E_{\text {mode }}(x, y)=\sum_{n} \beta_{n}^{-1 / 2} c_{n}^{-} e^{-i \beta_{n} y} u_{n}(x),
$$

where $c_{n}^{-}$is the amplitude of the $n$th downward propagating transmitted mode, and $\beta_{n}^{-1 / 2}$ is the analogous normalization factor. The corresponding upward oriented field is written with $c^{-} \rightarrow c^{+}$and $-i \beta_{n} y \rightarrow i \beta_{n} y$. We equate $E_{\text {mode }}(x, y)$ and $E_{\mathrm{pw}}(x, y)$ at the boundary $(y=0)$ and substitute $u_{n}(x)=\Sigma_{p} J_{p n} e_{p}(x)$, after which the sums can be removed, leaving

$$
\chi^{-1 / 2}(r+\delta)=\mathbf{J} \boldsymbol{\beta}^{-1 / 2} c^{-},
$$

where $\mathbf{J}$ performs the change of basis. Similarly, writing $e_{p}(x)=\Sigma_{n} \bar{J}_{n p} u_{n}(x)$ and taking a spatial derivative with respect to $y$ reveals

$$
\mathbf{J}^{\dagger} \boldsymbol{\chi}^{1 / 2}(r-\delta)=-\boldsymbol{\beta}^{1 / 2} c^{-},
$$

which represents the continuity of the normal field derivative across the boundary, where the dagger $\left({ }^{\dagger}\right)$ is the Hermitian transpose. In both these expressions, $\boldsymbol{\beta}$ and $\boldsymbol{\chi}$ represent square matrices with $\beta_{n}$ and $\chi_{p}$ on the diagonals. That is, $\boldsymbol{\beta}=\operatorname{diag}\left\{\beta_{n}\right\}$ and $\boldsymbol{\chi}=\operatorname{diag}\left\{\chi_{p}\right\}$. Taking care to preserve the ordering of terms since matrix multiplication is non-commutative, $c^{-}$may be eliminated, resulting in

$$
\delta+r=\boldsymbol{\chi}^{1 / 2} \mathbf{J} \boldsymbol{\beta}^{-1 / 2} \boldsymbol{\beta}^{-1 / 2} \mathbf{J}^{\dagger} \boldsymbol{\chi}^{1 / 2}(\delta-r) .
$$

For brevity, let

$$
\begin{aligned}
& \mathbf{A}=\boldsymbol{\chi}^{1 / 2} \mathbf{J} \boldsymbol{\beta}^{-1 / 2}, \\
& \mathbf{B}=\boldsymbol{\beta}^{-1 / 2} \mathbf{J}^{\dagger} \boldsymbol{\chi}^{1 / 2},
\end{aligned}
$$

where $\mathbf{A}$ and $\mathbf{B}$ are not to be confused with the eigenvector components $A$ and $B$ used previously. Then $\delta+r$ $=\mathbf{A B}(\delta-r)$, or

$$
r=(\mathbf{A B}+\mathbf{I})^{-1}(\mathbf{A B}-\mathbf{I}) \delta=\mathbf{R}_{12} \delta,
$$

i.e.,

$$
\mathbf{R}_{12}=(\mathbf{A B}+\mathbf{I})^{-1}(\mathbf{A B}-\mathbf{I})
$$

Similarly,

$$
c^{-}=\mathbf{B}(\delta-r)=\mathbf{B}\left(\delta-\mathbf{R}_{12} \delta\right)=\mathbf{B}\left(\mathbf{I}-\mathbf{R}_{12}\right) \delta,
$$

i.e.,

$$
\mathbf{T}_{12}=\mathbf{B}\left(\mathbf{I}-\mathbf{R}_{12}\right)=2 \mathbf{B}(\mathbf{A} B+\mathbf{I})^{-1} .
$$

The corresponding 21 terms (covering scattering from modes to modes and modes to orders) may be derived analogously. All together, there are four scattering matrices linking order and modal fields, in phase and amplitude, on either side of the interface:

$$
\begin{gathered}
\mathbf{R}_{12}=(\mathbf{A B}+\mathbf{I})^{-1}(\mathbf{A B}-\mathbf{I}), \\
\mathbf{R}_{21}=(\mathbf{B A}+\mathbf{I})^{-1}(\mathbf{I}-\mathbf{B A}), \\
\mathbf{T}_{12}=2 \mathbf{B}(\mathbf{A B}+\mathbf{I})^{-1},
\end{gathered}
$$

$$
\mathbf{T}_{21}=2 \mathbf{A}(\mathbf{B A}+\mathbf{I})^{-1}
$$

with

$$
\begin{aligned}
& \mathbf{A}=\boldsymbol{\chi}^{1 / 2} \mathbf{J} \boldsymbol{\beta}^{-1 / 2}, \\
& \mathbf{B}=\boldsymbol{\beta}^{-1 / 2} \mathbf{J}^{\dagger} \chi^{1 / 2} .
\end{aligned}
$$

\section{APPENDIX C: COMBINING FRESNEL SCATTERING MATRICES FOR FULL GRATING DERIVATION}

We now extend the mathematical description to a finite grating surrounded by a homogeneous medium. To completely describe the field above, below, and within the grating we use five fields $\left(r, c^{-}, c^{+}, t, \delta\right)$ as shown in Fig. 19. In addition to previously defined fields, we add $c^{+}$, a vector of complex upward propagating mode amplitudes reflected from the bottom surface, and $t$, a vector of complex plane-wave amplitudes transmitted through the grating. All five are linked by equations describing reflection and transmission at each interface:

$$
\begin{gathered}
r=\mathbf{R}_{12} \delta+\mathbf{T}_{21} \mathbf{P} c^{+}, \\
c^{-}=\mathbf{T}_{12} \delta+\mathbf{R}_{21} \mathbf{P} c^{+}, \\
c^{+}=\mathbf{R}_{21} \mathbf{P} c^{-}, \\
t=\mathbf{T}_{21} \mathbf{P} c^{-},
\end{gathered}
$$

where the thickness of the grating $t_{g}$ is incorporated via the complex matrix

$$
\mathbf{P}=e^{i \boldsymbol{\beta} t_{g}}, \quad \boldsymbol{\beta}=\operatorname{diag}\left\{\beta_{n}\right\},
$$

which propagates phase and amplitude of grating modes from one interface to the other, accounting for vertical propagation.

As $\mathbf{P}$ is included only now in the calculation, the parameter can be varied with little computational cost compared to the incident angle. In particular, the thickness of the grating as modeled can be varied without recalculating modes or overlap integrals; grating-air interface scattering matrices remain unchanged.

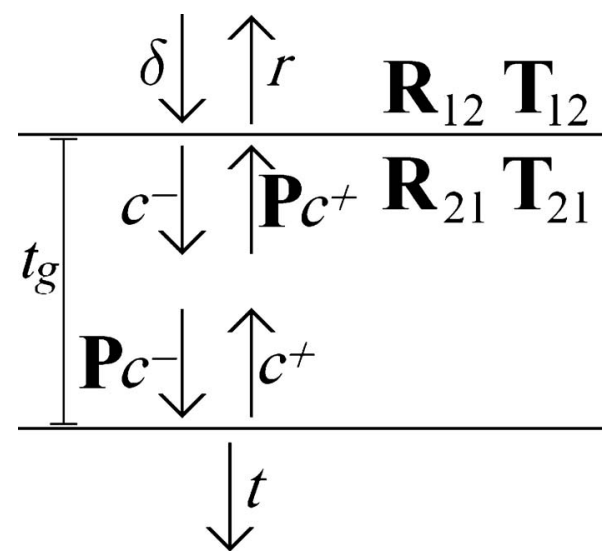

Fig. 19. Diagram showing the interaction of modes $\left(c^{ \pm}\right)$and orders $(\delta, r, t)$ within a complete grating formulation. 
These equations may be freely rearranged in a manner similar to the interface scattering matrices to provide the amplitudes of any given field as a function of the input field $\delta$. In particular

$$
\begin{gathered}
r=\left[\mathbf{R}_{12}+\mathbf{T}_{21} \mathbf{P} \mathbf{R}_{21} \mathbf{P}\left(\mathbf{I}-\mathbf{R}_{21} \mathbf{P} \mathbf{R}_{21} \mathbf{P}\right)^{-1} \mathbf{T}_{12}\right] \delta, \\
c^{-}=\left(\mathbf{I}-\mathbf{R}_{21} \mathbf{P} \mathbf{R}_{21} \mathbf{P}\right)^{-1} \mathbf{T}_{12} \delta, \\
c^{+}=\mathbf{R}_{21} \mathbf{P}\left(\mathbf{I}-\mathbf{R}_{21} \mathbf{P} \mathbf{R}_{21} \mathbf{P}\right)^{-1} \mathbf{T}_{12} \delta, \\
t=\mathbf{T}_{21} \mathbf{P}\left(\mathbf{I}-\mathbf{R}_{21} \mathbf{P} \mathbf{R}_{21} \mathbf{P}\right)^{-1} \mathbf{T}_{12} \delta .
\end{gathered}
$$

From these the full single grating scattering matrices $\mathbf{R}$ and $\mathbf{T}$, connecting plane waves above and below, may be read off:

$$
\begin{gathered}
\mathbf{R}=\mathbf{R}_{12}+\mathbf{T}_{21} \mathbf{P} \mathbf{R}_{21} \mathbf{P}\left(\mathbf{I}-\mathbf{R}_{21} \mathbf{P} \mathbf{R}_{21} \mathbf{P}\right)^{-1} \mathbf{T}_{12} \\
\mathbf{T}=\mathbf{T}_{21} \mathbf{P}\left(\mathbf{I}-\mathbf{R}_{21} \mathbf{P R} \mathbf{R}_{21} \mathbf{P}\right)^{-1} \mathbf{T}_{12}
\end{gathered}
$$

\section{ACKNOWLEDGMENT}

We gratefully acknowledge the financial support of the Australian Research Council through its Centre of Excellence and Discovery Grant Schemes.

\section{REFERENCES}

1. R. W. Wood, "The echelette grating for the infrared," Philos. Mag. 20, 770-778 (1910).

2. J. B. Pendry, "Negative refraction makes a perfect lens," Phys. Rev. Lett. 85, 183966 (2000).

3. Z. Jacob, L. V. Alekseyev, and E. Narimanov, "Optical hyperlens: Far-field imaging beyond the diffraction limit," Opt. Exp. 14, 8247-8256 (2006).

4. S. W. Hell and J. Wichmann, "Breaking the diffraction resolution limit by stimulated emission: Stimulated-emissiondepletion fluorescence microscopy," Opt. Lett. 19, 780-782 (1994).

5. X. Zhuang, "Nano-imaging with STORM," Nat. Photonics 3, 365-367 (2009).

6. P. C. Chaumet, K. Belkebir, and A. Sentenac, "Superresolution of three-dimensional optical imaging by use of evanescent waves," Opt. Lett. 29, 2740-2742 (2004).

7. K. Belkebir, P. C. Chaumet, and A. Sentenac, "Superresolution in total internal reflection tomography," J. Opt. Soc. Am. A 22, 1889-1897 (2005).

8. A. Sentenac, P. Chaumet, and K. Belkebir, "Beyond the Rayleigh criterion: Grating assisted far-field optical diffraction tomography," Phys. Rev. Lett. 97, 243901 (2006).

9. G. Maire, F. Drsek, J. Girard, H. Giovannini, A. Talneau, D. Konan, K. Belkebir, P. C. Chaumet, and A. Sentenac, "Experimental demonstration of quantitative imaging beyond Abbe's limit with optical diffraction tomography," Phys. Rev. Lett. 102, 213905 (2009).
10. C. J. Handmer, C. M. de Sterke, R. C. McPhedran, L. C. Botten, M. J. Steel, and A. Rahmani, "Blazing evanescent grating orders: A spectral approach to beating the Rayleigh limit," Opt. Lett. 35, 2846-2848 (2010).

11. A. Sentenac and P. Chaumet, "Subdiffraction light focusing on a grating substrate," Phys. Rev. Lett. 101, 013901 (2008).

12. L. Li, "New formulation of the Fourier modal method for crossed surface-relief gratings," J. Opt. Soc. Am. A 14, 2758-2767 (1997)

13. G. H. Derrick and R. C. McPhedran, "Coated crossed gratings," J. Opt. (Paris) 15, 69-81 (1984).

14. L. C. Botten, R. C. McPhedran, J. L. Adams, J. Andrewartha, and M. S. Craig, "The dielectric lamellar diffraction grating," Opt. Acta 28, 413-428 (1981).

15. L. C. Botten, M. S. Craig, R. C. McPhedran, J. L. Adams, and J. Andrewartha, "The finitely-conducting lamellar diffraction grating," Opt. Acta 28, 1087-1102 (1981).

16. L. C. Botten, M. S. Craig, and R. C. McPhedran, "Highly conducting lamellar diffraction gratings," Opt. Acta 28 1103-1106 (1981).

17. L. Li, "Multilayer modal method for diffraction gratings of arbitrary profile, depth, and permittivity,” J. Opt. Soc. Am. A 10, 2581-2591 (1993).

18. S. Campbell, L. C. Botten, R. C. McPhedran, and C. M. de Sterke, "Modal method for classical diffraction by slanted lamellar gratings,” J. Opt. Soc. Am. A 25, 2415-2426 (2008).

19. R. de L. Kronig and W. G. Penney, "Quantum mechanics of electrons in crystal lattices," Proc. R. Soc. London, Ser. A 130, 499-513 (1931).

20. U. Fano, "Effects of configuration interaction on intensities and phase shifts," Phys. Rev. 124, 1866-1878 (1961).

21. M. C. Hutley, Diffraction Gratings (Academic, 1982).

22. R. Petit, Electromagnetic Theory of Gratings (Springer, 1980).

23. E. Popov, L. Mashev, and D. Maystre, "Theoretical study of the anomalies of coated dielectric gratings," J. Mod. Opt. 33, 607-619 (1986).

24. E. Abbe, "Beiträge zur Theorie des Mikroskops und der mikroskopischen Wahrnehmung," Arch. Mikrosc. Anat. Entwicklungsmech. 9, 413-418 (1873).

25. R. Gordon, A. G. Brolo, D. Sinton, and K. L. Kavanagh, "Resonant optical transmission through hole-arrays in metal films: Physics and applications," Laser Photonics Rev. 4, 311-335 (2010).

26. A. Roberts and R. C. McPhedran, "Power losses in highly conducting lamellar gratings," Opt. Acta 34, 511-538 (1988).

27. J. B. Andersen and V. V. Solodukhov, "Field behavior near a dielectric wedge," IEEE Trans. Antennas Propag. 26, 598602 (1978).

28. P. Gregory, Bayesian Logical Data Analysis for the Physical Sciences (Cambridge U. Press, 2005).

29. F. M. Huang, Y. Chen, F. J. G. de Abajo, and N. I. Zheludev, "Optical superresolution through super-oscillations," J. Opt. A, Pure Appl. Opt. 9, S285-S288 (2007).

30. M. Vellekoop and A. Mosk, "Universal optimal transmission of light through disordered materials," Phys. Rev. Lett. 101, 120601 (2008).

31. E. van Putten, I. Vellekoop, and A. Mosk, "Spatial amplitude and phase modulation using commercial twisted nematic LCDs," Appl. Opt. 47, 2076-2081 (2008). 